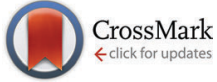

Cite this: Phys. Chem. Chem. Phys., 2015, 17, 22106

Received 8th June 2015,

Accepted 4th August 2015

DOI: $10.1039 / c 5 c p 03321 g$

www.rsc.org/pccp

\section{Tunable conduction type of solution-processed germanium nanoparticle based field effect transistors and their inverter integration $\dagger$}

\author{
Zeynep Meric, $\ddagger^{\mathrm{a}}$ Christian Mehringer, $\ddagger^{\mathrm{b}}$ Nicolas Karpstein, ${ }^{a}$ Michael P. M. Jank, ${ }^{\star c}$ \\ Wolfgang Peukert ${ }^{\mathrm{b}}$ and Lothar Frey ${ }^{\mathrm{ac}}$
}

\begin{abstract}
In this work we demonstrate the fabrication of germanium nanoparticle (NP) based electronics. The whole process chain from the nanoparticle production up to the point of inverter integration is covered. Ge NPs with a mean diameter of $33 \mathrm{~nm}$ and a geometric standard deviation of 1.19 are synthesized in the gas phase by thermal decomposition of $\mathrm{GeH}_{4}$ precursor in a seeded growth process. Dispersions of these particles in ethanol are employed to fabricate thin particulate films (60 to $120 \mathrm{~nm}$ in thickness) on substrates with a pre-patterned interdigitated aluminum electrode structure. The effect of temperature treatment, polymethyl methacrylate encapsulation and alumina coating by plasma-assisted atomic layer deposition (employing various temperatures) on the performance of these layers as thin film transistors (TFTs) is investigated. This coating combined with thermal annealing delivers ambipolar TFTs which show an $I_{\text {on }} / I_{\text {off }}$ ratio in the range of $10^{2}$. We report fabrication of n-type, $\mathrm{p}$-type or ambipolar Ge NP TFTs at maximum temperatures of $450{ }^{\circ} \mathrm{C}$. For the first time, a circuit using two ambipolar TFTs is demonstrated to function as a NOT gate with an inverter gain of up to 4 which can be operated at room temperature in ambient air.
\end{abstract}

In the emerging field of printable electronics dispersions of nanoparticles are seen as possible starting materials for new low cost device fabrication techniques like roll-to-roll processing. ${ }^{1,2}$ Examples of suitable materials and devices include e.g. a composite dispersion from indium tin oxide (ITO) NPs and conducting polymer NPs (3,4-ethylenedioxythiophene/ polystyrene sulfonic acid). This dispersion is used for transparent NP based electrode fabrication. ${ }^{3}$ Further, zinc oxide nanoparticles deposited from dispersion and post processed by a laser treatment can be utilized for NP TFT fabrication. ${ }^{4,5}$ Silicon ( $\mathrm{Si}$ ) can also be utilized in form of nanoparticles to fabricate TFTs as e.g. demonstrated by Weis et al. ${ }^{6}$ and Holman et $a l^{7}$ In this work we focus on Ge, because it is seen as a promising material for NP based device fabrication

\footnotetext{
${ }^{a}$ Chair of Electron Devices, Friedrich-Alexander-Universität Erlangen-Nürnberg, Cauerstrasse 6, 91058 Erlangen, Germany. E-mail: zeynep.meric@leb.eei.uni-erlangen.de, nicolas.karpstein@leb.eei.uni-erlangen.de, lothar.frey@leb.eei.uni-erlangen.de

${ }^{b}$ Institute of Particle Technology, Friedrich-Alexander-Universität Erlangen-Nürnberg, Cauerstrasse 4, 91058 Erlangen, Germany. E-mail: christian.mehringer@fau.de, wolfgang.peukert@fau.de

${ }^{c}$ Fraunhofer Institute for Integrated Systems and Device Technology, Schottkystrasse 10, 91058 Erlangen, Germany. E-mail: Michael.Jank@iisb.fraunhofer.de;

Fax: +49 9131 761-360; Tel: +49 9131 761-161

$\dagger$ Electronic supplementary information (ESI) available: Additions on NP morphology, FTIR, XRD and electronic device characterization. See DOI: 10.1039/c5cp03321g.

\# These authors contributed equally.
}

due to high bulk charge carrier mobilities (electron $\approx$ $3900 \mathrm{~cm}^{2} \mathrm{~V}^{-1} \mathrm{~s}^{-1}$, hole $\left.\approx 1900 \mathrm{~cm}^{2} \mathrm{~V}^{-1} \mathrm{~s}^{-1}\right)^{8}$ and the prospect for processing at lower temperatures. The formation of Ge NPs has been demonstrated by liquid phase synthesis. ${ }^{9,10}$ However, aerosol synthesis techniques are generally better suited for delivering high purity nanoparticles in a continuous and scalable process. ${ }^{11}$ Especially non-thermal plasma synthesis delivers high quality Ge NPs. ${ }^{12,13}$ As demonstrated by Holman et al. such Ge NPs can be deposited as thin films from the aerosol phase directly onto a substrate via an impactor, or by fabricating a NP dispersion and depositing it onto a substrate. ${ }^{14,15}$ In this fashion the fabrication of functional thin-film field-effect transistors (FETs) from dispersions of those NPs is possible. ${ }^{7}$ Additionally to the pure material properties it was already shown - for doped Si NPs for example that the termination of the particle surfaces influences the device performance. ${ }^{16}$ Practically, layer encapsulation can stabilize the particle film against environmental effects and define interfaces influencing the charge carrier concentration within nanoparticulate layers and consequently the device performance as has been demonstrated for Si $\mathrm{NPs}^{6}$ and zinc oxide NPs. ${ }^{17}$ By intentionally exploiting such surface effects, devices with different conduction types can be achieved utilizing only one starting material and processing step. The conduction type is then controlled by the post-processing parameters like temperature and the interface with the encapsulation material. 
Cost intensive conventional doping techniques like ion implantation or diffusion could thus be avoided.

Therefore, the aim of this work is to identify the impact of different layer encapsulations on the electrical performance of germanium nanoparticle layers and TFT set-ups. The device fabrication is based on Ge nanoparticles synthesized by a hotwall reactor aerosol process. For this, we utilize a two stage hotwall reactor (HWR) set-up for germane $\left(\mathrm{GeH}_{4}\right)$ pyrolysis. The first HWR is used for seeding the particle growth in the second HWR, which is mandatory for the NP quality. ${ }^{18}$ The as-produced particles are dispersed in ethanol inside a glove box system and spin coated onto substrates with a pre-patterned interdigitated aluminum electrode structure. Further processing is done by hot-plate annealing, polymethyl methacrylate coating or plasma-assisted atomiclayer deposition (ALD) of aluminum oxide. The influences of the film morphology and the post processing treatments on the device performance are investigated. We demonstrate the fabrication of n-type, p-type as well as ambipolar thin film FETs from the same Ge NP starting material solely depending on the post processing scheme. Ultimately, inverter integration is demonstrated utilizing two ambipolar thin film FETs from solution processed Ge nanoparticles operating in ambient atmosphere for the first time.

\section{Results and discussion}

Seeding ${ }^{19}$ is performed to synthesize the Ge NPs utilized in this study. It is mandatory to produce well-defined particles with a reproducible narrow size distribution. Constant $\mathrm{SiH}_{4}$ and $\mathrm{Ar}$ volume flow rates are fed into HWR I for this purpose and the resulting Si seeding aerosol ${ }^{18}$ is fed into HWR II where $\mathrm{GeH}_{4}$ is added at $1000{ }^{\circ} \mathrm{C}$ (see Experimental methods). For details about the morphological effect of the seeding please refer to the ESI $\dagger$ (cf. Fig. $\mathrm{S} 1)$.

The resulting Ge NPs show a particle size distribution (PSD) with a primary particle size around $33 \mathrm{~nm}$ and a geometric standard deviation of 1.19 as depicted in Fig. 1a. The diffractogram ( $c f$. Experimental methods section) of the powder is shown in Fig. 1b.
The diffractogram is best fit with a Ge-Si alloy containing exactly 100 at\% Ge utilizing the software Topas ( $c f$. Fig. S2, ESI $\dagger$ ), i.e. pure Ge NPs can be synthesized. The Si content resulting from the seeds must be very small, because X-ray diffraction (XRD) is very sensitive towards the lattice constant and thus the composition. The crystallite size can be estimated from the diffractogram to be around $33 \mathrm{~nm}$. This value is in excellent agreement with the PSD, meaning that the particles are nearly monocrystalline. Gas adsorption yields a size of $46 \mathrm{~nm}$, which is in reasonable agreement. The discrepancy may be due to water adsorption because the samples are not heat treated prior to measurement. It was found that a heat treatment in the case of Ge NPs drastically reduced the specific surface area. This can be explained by the instability of germanium oxide, which is known to undergo complex oxidation paths during thermal annealing. ${ }^{20-22}$ This powder is used for dispersion fabrication, thin film formation and device characterization.

Dispersion formation is performed in the glove box as described in the Experimental methods section. Ethanol is identified as suitable solvent in a solvent screening also involving different particle species which will be covered in a separate publication elsewhere. The result of the dynamic light scattering (DLS) measurements of a diluted dispersion sample of the Ge NPs in ethanol is shown in Fig. 2. The number density distribution is mono modal with a mean diameter of 63 to $71 \mathrm{~nm}$, depending on the measurement series (only one shown). This means the primary particles are forming aggregates in the dispersion. A small fraction of even bigger agglomerates of $200 \mathrm{~nm}$ in size becomes visible in the volume density distribution. However, the original dispersions are stable for several days and any sediment forming after longer storage times can easily be redispersed by ultrasonic treatment. Thus it is possible to create intrinsically stable dispersions in ethanol for application in thin film deposition.

Thin film deposition is carried out using dispersions of $1 / 3 \mathrm{wt} \%$ and $1 \mathrm{wt} \%$ of Ge NPs in ethanol. The rotational speed is set to $2000 \mathrm{rpm}$ and the number of spin coating cycles is varied from two to five. The top-down Scanning Electron Microscopy (SEM) image in Fig. 3a shows an example for a layer from a $1 / 3 \mathrm{wt} \%$ dispersion utilizing 3 deposition steps. It shows a low
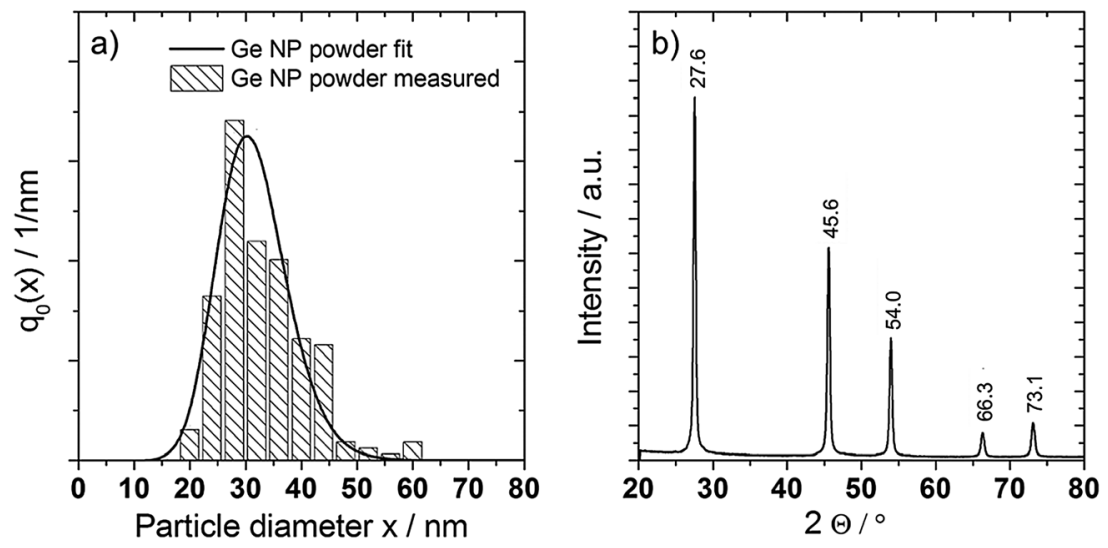

Fig. 1 (a) PSD of the Ge NP powder sample with a geometric standard deviation of 1.19; (b) diffractogram of the powder sample. 

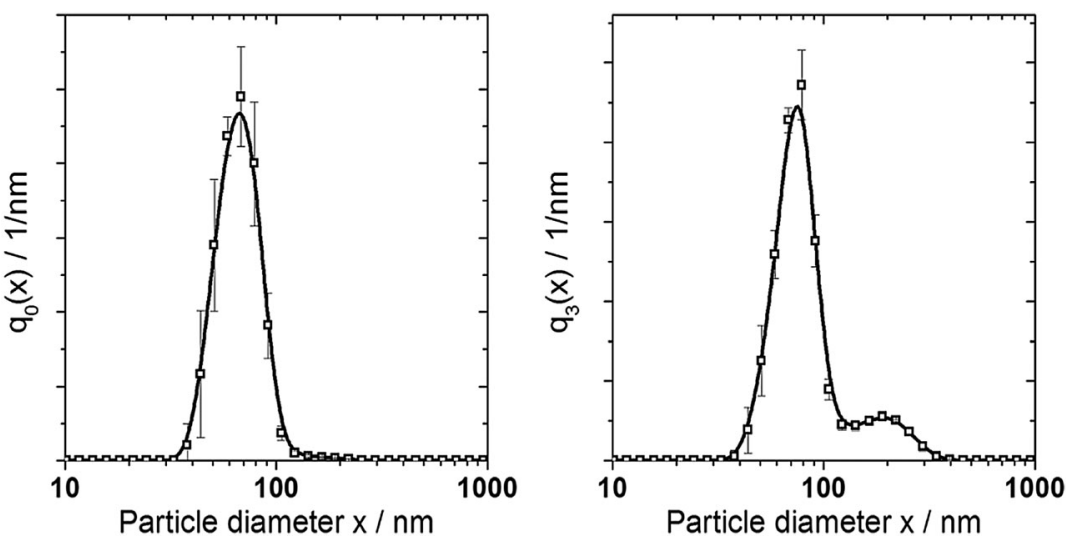

Fig. 2 Number and volume density distribution of the Ge NP powder sample dispersed in ethanol.
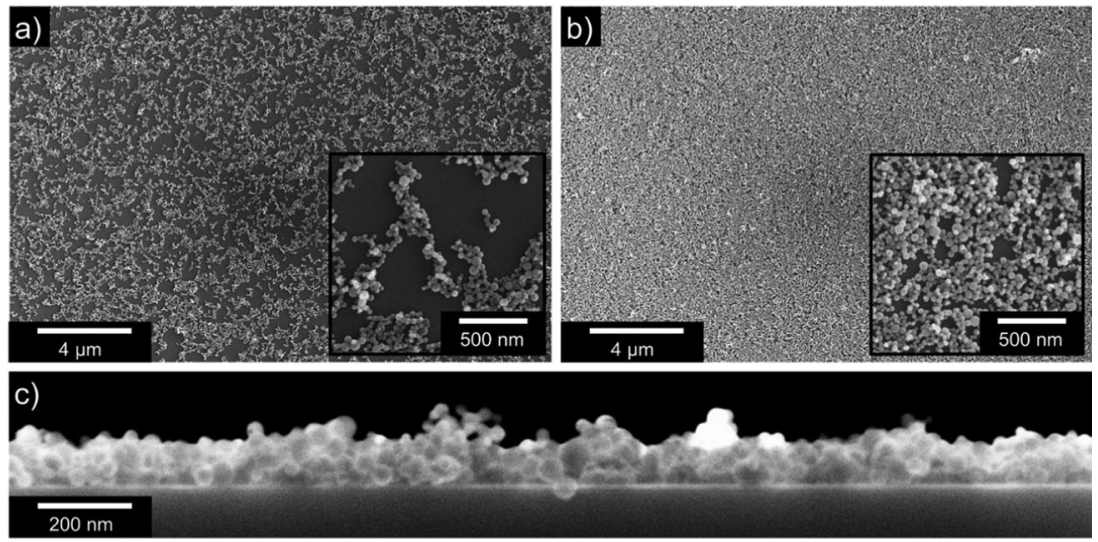

Fig. 3 (a) Porous Ge NP layer (1/3 wt\%, 3 deposition steps); (b) dense Ge NP layer (1 wt\%, 4 deposition steps). Insets show higher magnification. (c) Cross-sectional view of layer shown in $b$

coverage of the substrate surface with particles and consequently only very low percolation density. It is thus considered as a porous layer. Fig. $3 \mathrm{~b}$ shows a layer resulting from a $1 \mathrm{wt} \%$ dispersion deposited by spin coating 4 times. It shows a considerable higher coverage and is an example for a dense layer with a high percolation path density. For this layer also a crosssectional view is shown (Fig. 3c).
The film morphology is evaluated by SEM image analysis delivering an average film thickness and projected areal particle coverage of the substrate ( $c f$. Experimental methods section). The results are shown in Fig. 4 . The film thickness evaluation shows increasing film thickness from two to four deposition steps for the $1 \mathrm{wt} \%$ dispersion and from two to three deposition steps for the $1 / 3 \mathrm{wt} \%$ dispersion. Applying a higher number of deposition
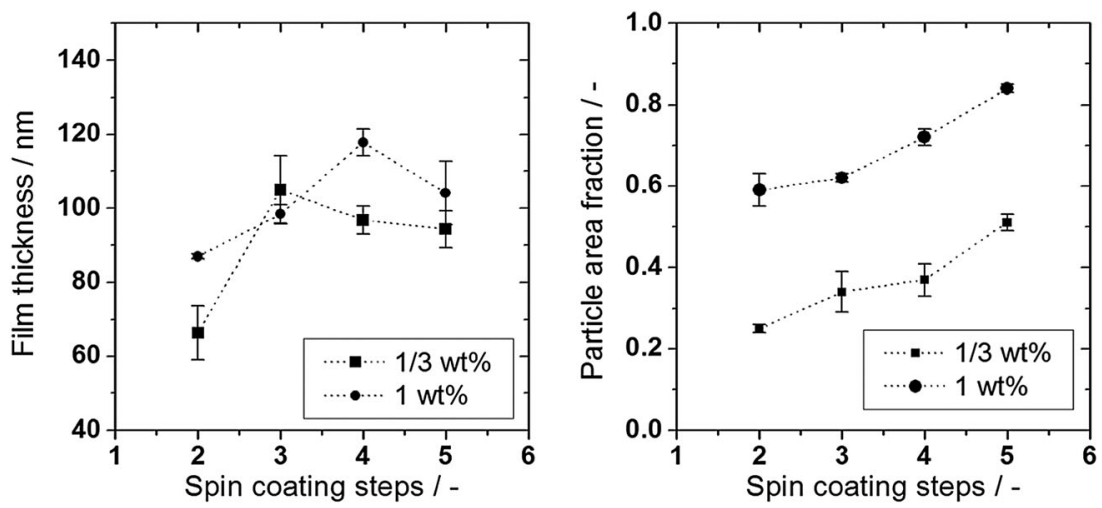

Fig. 4 Film thickness and particle area fraction for layers prepared from $1 / 3 \mathrm{wt} \%$ and $1 \mathrm{wt} \%$ Ge NP powder in ethanol at $2000 \mathrm{rpm}$ and varying spin coating steps. 
steps leads to decreasing film thicknesses in both cases. Generally the film thickness ranges between 60 and $120 \mathrm{~nm}$. A very clear trend is observed for the Particle Area Fraction (PAF), which is in essence a projected surface coverage, determined by SEMsupported image analysis ( $c f$. Experimental methods). For both dispersions the PAF increases with increasing number of deposition steps. However, the films do not get thicker necessarily. Although particle material added by consecutive deposition steps is incorporated into empty sites in the preexisting film, loosely bound portions of the film are removed from the surface and transported off the substrate by rotational forces. The same is found for material from preceding steps, which is redispersed and then redeposited or transported off the substrate. Subsequent iterations lead to significant film compaction and even thinning. The fact that thinning occurs even at lower counts of spinning cycles for dispersions having lower particle load strengthens this hypothesis. Loosely bound particles may easily be washed away by subsequent deposition steps whereas new material deposited can fill voids in the existing film, resulting in an improvement of the interface and film density ( $c f$. Fig. 3). Maximum interface coverage in combination with a low thickness of the film is crucial for achieving high ON currents at lowest OFF currents in a TFT. Concerning this matter, an optimum (with respect to the performed experiments) is found in the layer prepared from $1 \mathrm{wt} \%$ dispersion at three spin coating steps which will be discussed later. Compared to the morphology findings this is reasonable, because a good percolation density at the semiconductor-dielectric interface is achieved at still a very thin layer.

The electrical characteristics of Ge NP thin-films were evaluated in TFT test structures. The output characteristics of TFT structures prepared from $1 \mathrm{wt} \%$ dispersion with different numbers of deposition steps are depicted in Fig. 5a. All films have been annealed for $15 \mathrm{~min}$ at $450{ }^{\circ} \mathrm{C}$ on a hot plate (HP). Upon annealing in $\mathrm{N}_{2}$ atmosphere, significant drain currents are observed showing gating by externally applied fields while as-deposited layers show low current levels with minimal gating ( $c f$. Fig. S3, ESI $\dagger$ ). We attribute this change in conductivity to two effects depicted in detail in the $\mathrm{ESI} \dagger$ ( $c f$. Fig. S4). Firstly, a purification of the particle surfaces is achieved by the removal of organic residuals originating from solvent in the dispersion and water from the NP surface, resulting in lower energetic barriers for the charge transfer between particles. Secondly, the surface termination of the particles changes form mostly hydrogen-terminated (as prepared) to mostly hydroxylterminated (annealed). This kind of termination is believed to act as electron donating. ${ }^{14}$ A morphology change due to the annealing is not observed, as demonstrated by representative SEM images in the ESI $\dagger$ ( $c f$. Fig. S5). A higher gate-source voltage leads to a higher drain current indicating an n-type conduction mechanism with the formation of an electron channel at the interface between the particle layer and the dielectric. The output characteristics lack the specific features that are usually associated with a TFT. Instead of showing typical triode and saturation regions, the drain-current follows a power-law $I \sim V^{m}$. This behavior is usually observed for space-charge-limited-currents (SCLC) ${ }^{23}$ where carriers trapped in the poorly conducting bulk limit further injection. A similar behavior could be observed for Si NP layers produced with the same synthesis method. ${ }^{6}$ For the Ge NP layers investigated in this study, the exponents $m$ which were determined from the slope of log-log output curves are well below 2 after annealing which indicates a reduction of the trap-state density in the layer ( $c f$. Fig. S6, ESI $\dagger$ ). We assume that device characteristics are the result of a superposition of the SCLC in the bulk film and field effect induced current in the channel at the interface of the dielectric.

The optimum particle layer with respect to a maximum $I_{\mathrm{on}} / I_{\mathrm{off}}$ ratio is found to be from $1 \mathrm{wt} \%$ dispersion and three deposition steps confirming the morphological data. The transfer characteristics of the optimum layer is shown in Fig. $5 \mathrm{~b}$ in comparison to the layer that shows the highest PAF value. Although the drain current for five times deposited sample is higher (Fig. 5a), the $I_{\text {on }} / I_{\text {off }}$ ratio is higher for the three times deposited sample (Fig. 5b). In the case of the five times deposited layer, a higher contribution of the bulk of the film to the drain current is expected due to a larger number of percolation paths. This leads to a higher off-current since the current in the bulk is not affected by the gate voltage. In that sense, the optimum layer with respect to a high $I_{\text {on }} / I_{\text {off }}$ is the three times deposited layer. It shows an $I_{\text {on }} / I_{\text {off }}$ ratio in the order of $10^{2}$ with a large hysteresis whereas for the sample with the higher PAF value
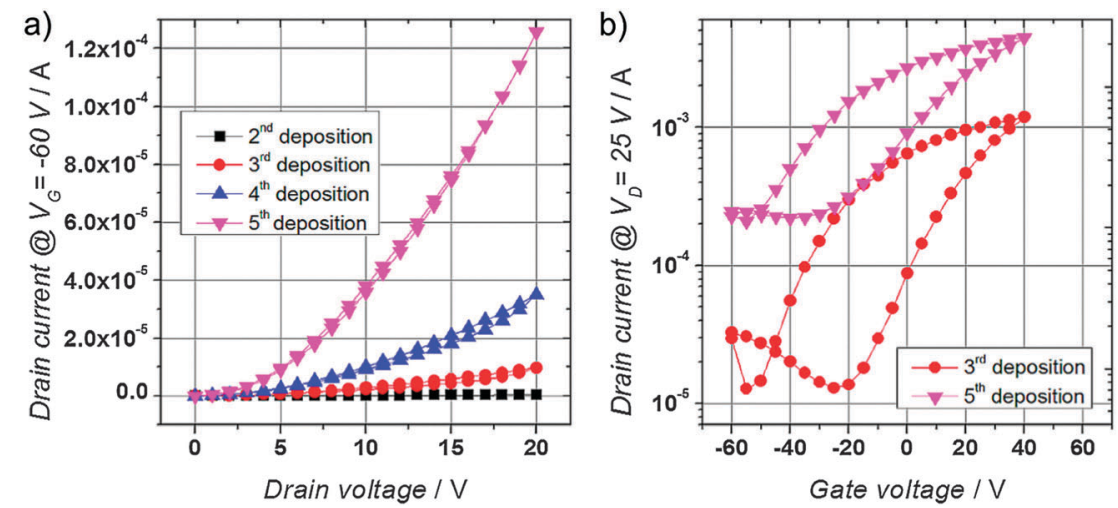

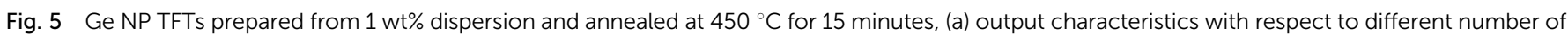
deposition steps, (b) transfer characteristics of 3 and 5 times deposited samples. 
the $I_{\mathrm{on}} / I_{\text {off }}$ ratio is one order of magnitude lower. Most likely the undesirable hysteresis is originating from trap states in the layer volume or at the semiconductor/dielectric interface ${ }^{24,25}$ and/or trapped charges induced by water molecules adsorbed on the semiconductor layer. ${ }^{6,26}$ Moreover, the high surface to volume ratio and the porous nature of the nanoparticle layers makes the surface chemistry of the nanoparticles become highly important with respect to the TFT device performance. ${ }^{7}$ For this reason, spin coating of polymethyl methacrylate and ALD of alumina as a passivation layer on top of the TFT devices are investigated.

The output and transfer characteristics of an optimized Ge NP TFT, annealed at $450{ }^{\circ} \mathrm{C}$ for 15 minutes prior to the polymethyl methacrylate (PMMA) encapsulation can be found in Fig. 6. For the output and transfer characteristics of the five times deposited sample see Fig. S7 (ESI $\dagger$ ). The device characteristics show the indirect effect of PMMA encapsulation on and within the porous nanoparticle layers. Covering both the particle surfaces and the open areas in the porous nanoparticle layer (i.e. the interface to $\mathrm{SiO}_{2}$ ) with PMMA clearly suppresses hysteresis and further improves the drain current with distinct response to different gate-source voltages (gating). This is most likely due to the reduction of surface trap states (e.g. dangling bonds ${ }^{27}$ ) and replacement of water adsorbents which are supposed to be responsible for the large hysteresis. ${ }^{6}$ Improvement in conduction also supports the hypothesis that more charge carriers are released from trapping sites after PMMA passivation. The device still exhibits n-type operation with $I_{\text {on }} / I_{\text {off }}$ ratios of about 100. The calculated field-effect mobility is in the range of $10^{-3} \mathrm{~cm}^{2} \mathrm{~V}^{-1} \mathrm{~s}^{-1}$ ( $c f$. Fig. S8 for the comparison between three times and five times deposited samples, ESI $\dagger$ ).

Atomic layer deposition enables the deposition of ultra-thin, uniform and conformal films with high step coverage and atomic controllability. ${ }^{28}$ Therefore, ALD deposition of alumina $\left(\mathrm{Al}_{2} \mathrm{O}_{3}\right)$ onto the nanoporous nanoparticle layers is investigated in order to form a passivation layer. Recently, the same approach was used to passivate germanium nanowires grown on gold nanoparticles. ${ }^{29}$ This study shows that thin $\mathrm{Al}_{2} \mathrm{O}_{3}$ can eliminate surface charge trapping and protect the germanium surface from the adsorption of ambient molecules, resulting in a current increase. More recently, the same approach for $\mathrm{ZnO}$ nanocrystals has been studied. ${ }^{17}$ Thimsen et al. showed the elimination of electronic traps on nanocrystal surfaces using ALD deposited $\mathrm{Al}_{2} \mathrm{O}_{3}$ and confirmed passivation against the adsorption of molecules from the ambient atmosphere.

For different ALD process temperatures similar improvements were observed. After ALD passivation, a change in conduction type from n-type to p-type behavior was observed (Fig. 7a). ${ }^{6}$ Additionally, an increase in conductivity and a suppression of the
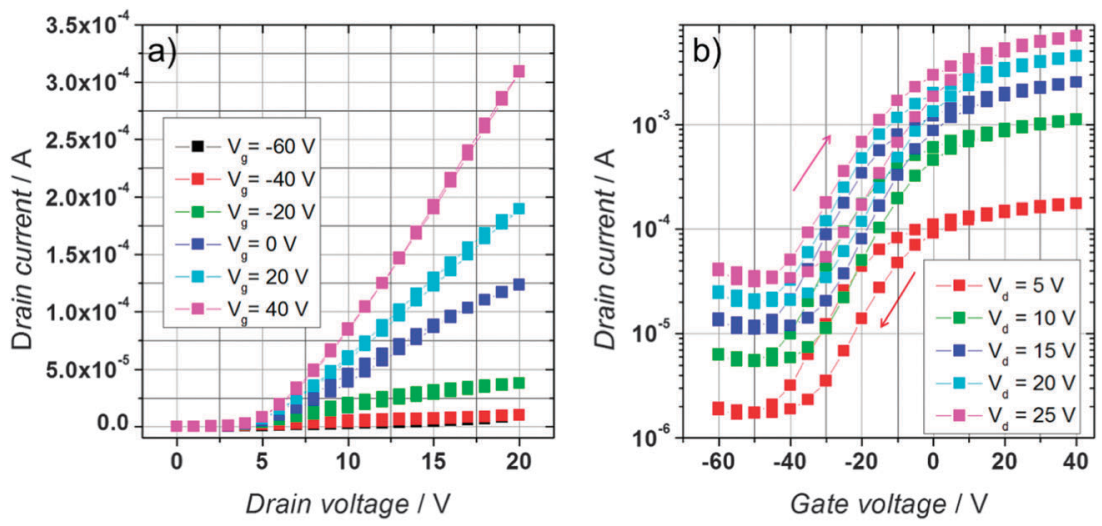

Fig. 6 Hot plate treated (at $450{ }^{\circ} \mathrm{C}$ for $15 \mathrm{~min}$ ) and PMMA encapsulated Ge NP TFTs ( $1 \mathrm{wt} \%$, three deposition steps) (a) output characteristics and (b) semi-log transfer characteristics.
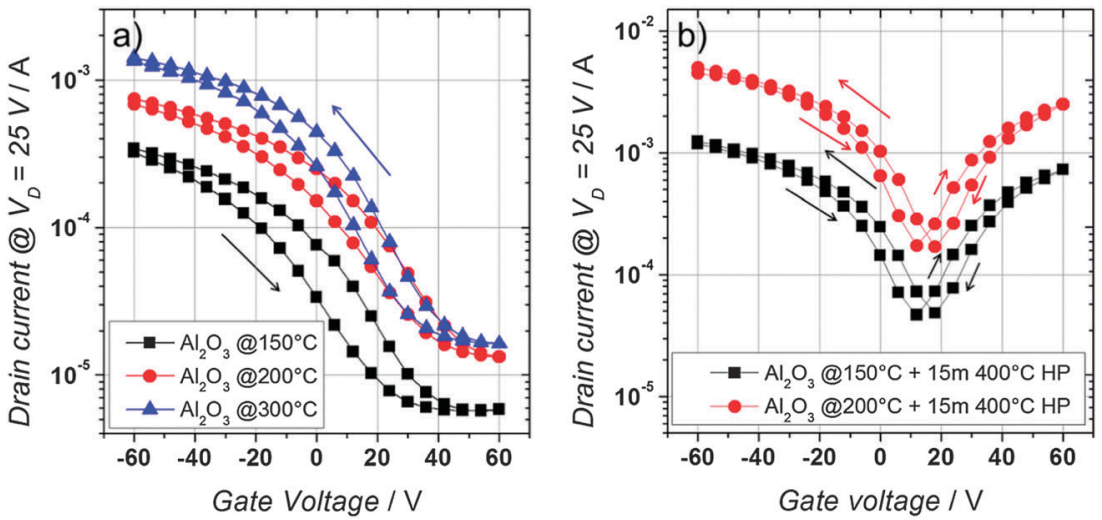

Fig. 7 (a) Semi-log transfer characteristics of reference device after $\mathrm{Al}_{2} \mathrm{O}_{3}$ deposition by ALD and (b) sequential hot plate annealing. 
hysteresis behavior, similar to the case of PMMA encapsulation was observed. Since the ALD process takes place at elevated temperatures, the samples were not annealed on a hot plate prior to the encapsulation. Apparently, the ALD encapsulation (which provides fixed negative charges) introduces shallow acceptor states at the particle surface that are overcompensating deep and shallow donor states. The $\mathrm{Al}_{2} \mathrm{O}_{3}$ encapsulation is also effective for passivation of surface dangling bonds and provides altered conductivity levels with $I_{\mathrm{on}} / I_{\text {off }}$ ratios of around 100 . The samples show a clear off-state (Fig. 7a, black curve) and the current levels are increasing with increasing ALD process temperature. The calculated field-effect mobilities for different ALD temperatures are in the range of $10^{-5}$ to $10^{-4} \mathrm{~cm}^{2} \mathrm{~V}^{-1} \mathrm{~s}^{-1}$. (cf. Fig. S9 for $5 \times$ deposited samples after ALD, ESI $\dagger$ )

After ALD passivation, we further investigated hot plate annealing in $\mathrm{N}_{2}$ atmosphere in the glove box (Fig. 7b). As a result of the treatment, the devices exhibit ambipolar $i-v$ characteristics with symmetric behavior around a gate voltage of about $18 \mathrm{~V}$, i.e. almost identical current levels in the n-type and the p-type branch of the characteristics. Similar results for Ge NC devices annealed at $500{ }^{\circ} \mathrm{C}$ by commercial rapid thermal annealing in vacuum with $\mathrm{N}_{2}$ flow were also reported previously by Holman et al. ${ }^{7}$ Devices delivering ambipolar $i-v$ characteristics have shown applicability to simple logic gates. ${ }^{30}$

Inverter integration of two TFTs for establishing a NOT gate/inverter (Fig. 8a) has been tested. For optimization of transfer characteristics, we select a lower mobility device (i.e. ALD@150 ${ }^{\circ} \mathrm{C}$, Fig. 7b) as the load transistor and the higher mobility device (ALD@200 ${ }^{\circ} \mathrm{C}$, Fig. 7b) as the driver transistor. The respective transfer curves depending on the supply voltage $V_{\mathrm{DD}}$ are depicted in Fig. 8b. The curves show the expected switching from high to low state with increasing input signal. For low voltages both TFTs are turned on in p-type operation and the output voltage is defined by the voltage divider between the two TFTs. Upon increasing the voltage, the driver TFT first reaches the transition point between $\mathrm{p}$ - and n-type operation. This point can be seen in the maximum close to $0 \mathrm{~V}$ in the inverter characteristics at $20 \mathrm{~V}$ and $30 \mathrm{~V}$ supply voltage. When increasing the input voltage further, the driver TFT is increasingly conducting in n-type operation whereas the load TFT, still p-type, sees an - in absolute values - decreasing gate-to-source voltage (i.e. difference between $V_{\mathrm{IN}}$ and $V_{\mathrm{DD}}$ ). The minimum output voltage is reached when the load TFT is at its maximum channel resistance which is seen in Fig. $8 \mathrm{~b}$ at gate voltages around $20 \mathrm{~V}$. Increasing the input voltage further will force both TFTs in n-type mode increasing the output voltage again. However this input condition is exceeding the supply voltage and thus only of minor interest. The curves show an optimum operation window at a supply voltage of around $30 \mathrm{~V}$ where the off state of the NOT gate reaches already $2 / 3$ of the supply voltage. Increasing the output voltage further leads to a limitation of the lowinput state to about $23 \mathrm{~V}$ (see transfer characteristics at $V_{\mathrm{DD}}$ of $40 \mathrm{~V}$ and $50 \mathrm{~V}$ in Fig. 8b). Also seen is a severe influence of hysteresis which should be further suppressed by proper engineering of the interfaces between the particles and the encapsulating layer.

The applicability of the presented devices in circuits is indeed promising as derived from the backward transfer curves which are plotted as solid symbols in Fig. 8b. The latches exhibit inverter gains of up to 4 with sufficiently high supply voltage which makes them applicable to fast switching circuits. Also chaining of the circuit elements is possible without fading dynamics, i.e. loss of on/off ratios. For example, the operation at a supply voltage of $30 \mathrm{~V}$ in our example would lead to stable high and low levels of $17.5 \mathrm{~V}$ and $7.5 \mathrm{~V}$ after 3 to 4 inverter stages even in the presented, not optimized configuration. Further improvements with respect to noise margins can be achieved by decreasing the $W / L$-ratio of the driver TFT, thus bringing up the output voltage significantly at low input voltages.

In summary, we have synthesized Ge NPs in the gas phase and fabricated solution-processed Ge NP TFTs. We studied the effect of different surface passivation and encapsulation techniques. As we could demonstrate, the surface chemistry plays an important role in terms of the device characteristics of nanoparticle based thin film transistors. By altering the NP surface we could successfully tailor the NP based device performance. While as-deposited layers from Ge NPs exhibit almost insulating properties, annealing introduces $\mathrm{OH}$ surface groups and removes residuals originating from the solvent. Consequently n-type conduction is observed. Furthermore, PMMA encapsulated devices show gating with improved conductivity levels and suppressed hysteresis with unchanged
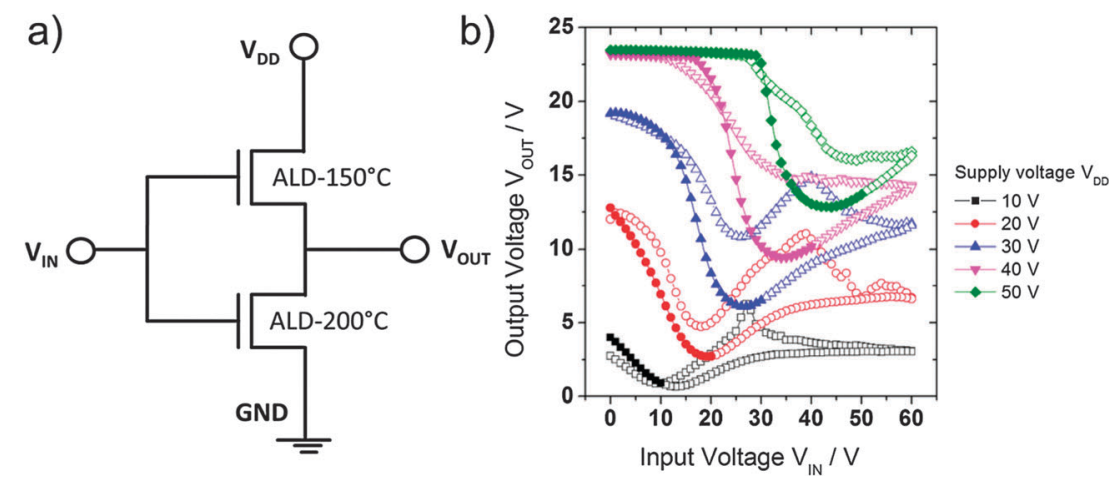

Fig. 8 (a) Circuit schematic and (b) transfer characteristics of the NOT gate derived from combination of a high mobility driver TFT derived from ALD encapsulation at $200{ }^{\circ} \mathrm{C}$ and a low-mobility load TFT derived from ALD encapsulation at $150{ }^{\circ} \mathrm{C}$. 
conduction type. ALD encapsulation leads to p-type and ambipolar behavior depending on whether further annealing is performed or not. Two ambipolar TFTs form a functional inverter set-up with a lower mobility device as load transistor and a higher mobility device as driver transistor. By a combination of surface chemistry alteration and thermal processing steps none of which exceeds a process temperature of $450{ }^{\circ} \mathrm{C}$, n-type, p-type or ambipolar Ge NP TFTs can be fabricated. To conclude, this work shows how surface chemistry can influence device performance additional to bulk material properties.

\section{Experimental methods}

The experimental two stage hot-wall reactor set-up for the particle synthesis used in this work is described in detail elsewhere. ${ }^{31}$ As described by Mehringer et al. ${ }^{18}$ for Ge-Si alloy NP synthesis, the first hot-wall reactor (HWR I) is used for seeding ${ }^{19}$ the second hot-wall reactor (HWR II) by silane $\left(\mathrm{SiH}_{4}\right)$ pyrolysis. To do so, $\mathrm{SiH}_{4}$ with a partial pressure of $0.042 \mathrm{mbar}$ in Argon (Ar) is fed into HWR I resulting in the seeding aerosol. It is found that seeding is possible between 900 and $1100{ }^{\circ} \mathrm{C}$ in HWR I. The particle morphology drastically changes if no seeding is applied ( $c f$. ESI $\dagger$ ). Between HWR I and HWR II the precursor $\mathrm{GeH}_{4}$ and Ar quench gas are added. The temperature in HWR II is set to $1000{ }^{\circ} \mathrm{C}$ and the $\mathrm{GeH}_{4}$ partial pressure is $0.30 \mathrm{mbar}$ at $25 \mathrm{mbar}$ total pressure. The resulting Ge NP powder is collected on a membrane filter. The latter is contained in a filter unit which can be flooded with Ar and sealed by two ball valves prior to detachment from the reactor set-up. In this manner the particles can be inserted into a nitrogen filled glove box system without contact to ambient conditions. The technique allows delivering powders into the glove box without the formation of a fully developed native oxide layer. However, we do not claim that the particle surface is atomically clean. SEM micrographs are taken with a Zeiss Gemini Ultra 55. Particle size analysis is performed with the software ImageJ. At least 150 particles are evaluated regarding their size for reliable statistics. Thin film formation is mainly characterized by two measures. The particle area fraction (PAF) is a projected surface coverage. It does not take into account a packing structure or height profile, but it is an indicator for the percolation path density. To determine the PAF, several top down SEM images of the prepared layers are converted into binary images, by defining a threshold on the grey scale of the respective image. ImageJ then returns a value for the black to white ratio, being 1-PAF, for every image. Averaging over at least three images delivers the PAF value for every prepared layer. Film thicknesses are determined in a similar way, starting from cross sectional SEM images of cleaved samples. The substrate is completely cropped from the picture, which is then converted to binary. The PAF determined is converted into a mean film thickness utilizing the total white area and dividing it by the total length of the picture. At least two pictures are averaged. This value does not take into account the roughness and density of the layer, but like the PAF value is a first order estimate for the comparison of differently prepared samples. One example for the PAF determination procedure is shown in Fig. 9a and b, the film thickness evaluation is exemplarily shown in Fig. 9c and d.

X-ray diffraction is conducted using a Bruker D8 Advance (Bragg-Brentano geometry) with $\mathrm{CuK} \alpha$-radiation (0.154 nm wavelength). A maximum of $30 \mathrm{~min}$ of air exposure precedes the measurement. Si single crystal containers (vicinal cut in [911]direction) cased in PMMA sample holders are employed for the powder sample. The powder surface is prepared as flat as possible. The Bragg-angle $(2 \theta)$ range is covered in a step size of $0.021^{\circ}$ with a measuring time of $2 \mathrm{~s}$ per step from $20^{\circ}$ to $80^{\circ}$. The evaluation is done with the software TOPAS (Bruker AXS). Peak fitting and simultaneous cell parameter refinement is performed with a quantitative Rietveld analysis, to determine the crystallite size and composition of the produced NPs. ${ }^{18}$ The measurement at the XRD device must be performed at ambient conditions. An interesting side note is the absence of $\mathrm{GeO}_{2}$ patterns, meaning that only a thin sub-stoichiometric (amorphous) GeO layer is formed around the particles upon air exposure for $30 \mathrm{~min}^{20,21}$ Longer air exposure leads to the formation of stoichiometric and crystalline $\mathrm{GeO}_{2}$, which can be recognized in the diffractograms (not shown). The stated crystallite size is a volume averaged column height calculated from the full width half maximum (FWHM). The method uses the Scherrer constant $\mathrm{k}$ at the default value of 0.89 for spheres (0.89 Vol-FWHM), although the use of a volume averaged column height which is calculated from the integral breadth (LVol-IB) is normally recommended. However, this recommendation is based on the assumption that the crystal shape is unknown, which is not the case in this work. Comparison of XRD analysis with SEM shows that in the analyzed samples most individual particles are
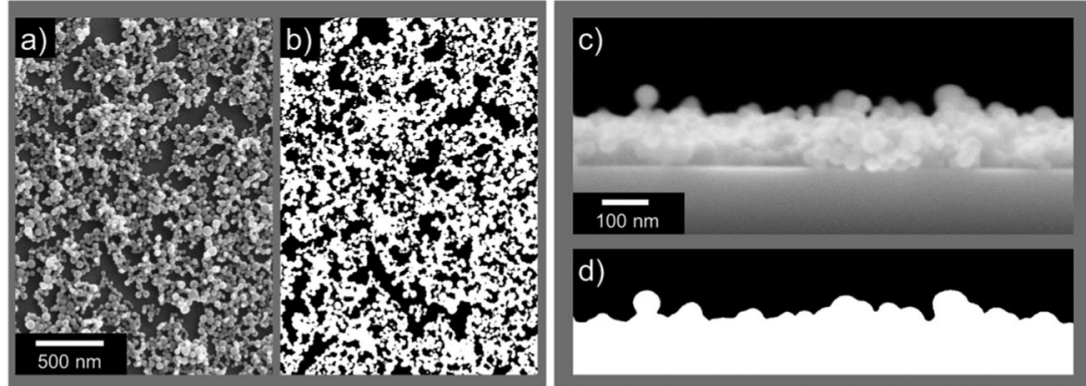

Fig. 9 Exemplary micrograph processing for particle area fraction determination and film thickness evaluation. 
of spherical shape and that the 0.89 Vol-FWHM values fit best compared to primary particle size. The analysis report can be found in the ESI $\dagger$ and also states LVol-IB. ${ }^{32}$ The specific surface area is investigated by gas adsorption measurements using a NOVA 4000 e gas sorption analyzer from Quantachrome instruments following the method of Brunauer, Emmet and Teller (BET). Measurements are conducted at $77 \mathrm{~K}$ (liquid nitrogen). The specific surface area is determined with a standard five point method. The specific surface area is converted into the Sauter diameter utilizing the density of bulk Ge. Dispersions with weight concentrations of $1 / 3 \mathrm{wt} \%$ and $1 \mathrm{wt} \%$ of Ge NPs in water free ethanol are prepared inside a glove box using an ultrasonic finger UP $100 \mathrm{H}$ by Hielscher at an amplitude of $100 \%$ and a cycle of 0.5 for 10 minutes. The ethanol absolute (anhydrous) is supplied by VWR and utilized as delivered. For DLS analysis, the $1 \mathrm{wt} \%$ dispersion is diluted to $1 / 250 \mathrm{wt} \%$ with pure solvent inside the glove box. DLS is performed with a Malvern Nano ZS instrument (Malvern Instruments GmbH, Germany) equipped with a $633 \mathrm{~nm}$ laser. The dispersion is filled into a Malvern dip cell kit inside the glove box to prevent oxidation of the particles. The dip cell is then encapsulated in the glove box and brought to the instrument. The measurements are performed immediately after opening the encapsulation and inserting the cell into the instrument. Three measurements are performed in a row and are averaged. The dispersions are utilized for thin film formation by applying them to substrates described below. This is accomplished by spin coating. The spin coater is a Delta6 RC BM by Süss Micro Tec integrated into the glove box. The dispersion is spin coated onto the substrate at $2000 \mathrm{rpm}$ two to five times. The process described by Weis et $a .^{6}$ is utilized. Substrates for electrical characterization are highly p-doped silicon wafers (for gate electrode) with a $200 \mathrm{~nm}$ thermally grown silicon dioxide layer (as dielectric). A prestructured interdigitated electrode structure for source and drain contacts made of aluminum is prepared as described by Weis et al. resulting in a width/length $(W / L)$ ratio of $50000 .^{6}$ In this manner TFT devices are produced by the spin coating the dispersions on top of the electrodes. A substrate schematic is shown in the ESI $\dagger$ (cf. Fig. S10). For film morphology evaluation substrates without metal electrodes are used. Encapsulation of the as-prepared TFTs with $30 \mathrm{~nm}$ of $\mathrm{Al}_{2} \mathrm{O}_{3}$ is performed using plasma-assisted atomiclayer deposition in a FlexAl system from Oxford Instruments at 15 mTorr $(1$ Torr $\approx 133 \mathrm{~Pa}) .^{6}$ The deposition is performed at 150 , 200 and $300{ }^{\circ} \mathrm{C}$. The samples are transferred in plastic sample containers wrapped in parafilm. PMMA encapsulation is performed like described by Weis et al. ${ }^{6}$ For the TFT device characterization two dual-channel source meter unit (SMU) Keithley 2636 by Keithley are used. The measurements were performed within the glove box system in an electromagnetically shielded box under the exclusion of light at room temperature. Transfer and output curves are generated using the commercial software provided by Keithley and an automated LabVIEW program.

\section{Conflict of interest}

The authors declare no competing financial interest.

\section{Acknowledgements}

The authors acknowledge the funding of the Deutsche Forschungsgemeinschaft (DFG) through the Cluster of Excellence "Engineering of Advanced Materials" at the FriedrichAlexander-Universität Erlangen-Nürnberg and the GRK 1161 Disperse Systems for Electronic Applications. We thank S. Weis and S. Polster from the chair of electron devices for fruitful discussion.

\section{Notes and references}

1 F. Erogbogbo, T. Liu, N. Ramadurai, P. Tuccarione, L. Lai, M. T. Swihart and P. N. Prasad, ACS Nano, 2011, 5(10), 7950-7959.

2 O. Yasar-Inceoglu and L. Mangolini, Mater. Lett., 2013, 101, 76-79.

3 I. Maksimenko, D. Kilian, C. Mehringer, M. Voigt, W. Peukert and P. J. Wellmann, J. Appl. Phys., 2011, 110(10), 104301.

4 H. Pan, N. Misra, S. H. Ko, C. P. Grigoropoulos, N. Miller, E. E. Haller and O. Dubon, Appl. Phys. A: Mater. Sci. Process., 2009, 94(1), 111-115.

5 M. Baum, JLMN, 2013, 8(2), 144-148.

6 S. Weis, R. Körmer, M. P. M. Jank, M. Lemberger, M. Otto, H. Ryssel, W. Peukert and L. Frey, Small, 2011, 7(20), 2853-2857.

7 Z. Holman, C.-Y. Liu and U. Kortshagen, Nano Lett., 2010, 10(7), 2661-2666.

8 S. Kim, B. Walker, S. Y. Park, H. Choi, S.-J. Ko, J. Jeong, M. H. Yun, J. C. Lee, D. S. Kim and J. Y. Kim, Nanoscale, 2014, 6(17), 10156.

9 E. J. Henderson, C. M. Hessel and J. G. C. Veinot, J. Am. Chem. Soc., 2008, 130(11), 3624-3632.

10 D. A. Ruddy, J. C. Johnson, E. R. Smith and N. R. Neale, ACS Nano, 2010, 4(12), 7459-7466.

11 F. Kruis, H. Fissan and A. J. Peled, Aerosol Sci., 1998, 29(5-6), 511-535.

12 P. Cernetti, R. Gresback, S. A. Campbell and U. Kortshagen, Chem. Vap. Deposition, 2007, 13(6-7), 345-350.

13 R. Gresback, Z. Holman and U. Kortshagen, Appl. Phys. Lett., 2007, 91(9), 093119.

14 Z. C. Holman and U. R. Kortshagen, Nanotechnology, 2010, 21(33), 335302.

15 Z. Holman and U. Kortshagen, Langmuir, 2009, 25(19), 11883-11889.

16 R. Gresback, N. J. Kramer, Y. Ding, T. Chen, U. R. Kortshagen and T. Nozaki, ACS Nano, 2014, 8(6), 5650-5656.

17 E. Thimsen, M. Johnson, X. Zhang, A. J. Wagner, K. A. Mkhoyan, U. R. Kortshagen and E. S. Aydil, Nat. Commun., 2014, 5, 5822 .

18 C. Mehringer, C. Kloner, B. Butz, B. Winter, E. Spiecker and W. Peukert, Nanoscale, 2015, 7(12), 5186-5196.

19 M. K. Alam and R. C. Flagan, Aerosol Sci. Technol., 1986, 5(2), 237-248.

20 A. Molle, M. N. K. Bhuiyan, G. Tallarida and M. Fanciulli, Appl. Phys. Lett., 2006, 89(8), 83504. 
21 G. Pourtois, M. Houssa, A. Delabie, T. Conard, M. Caymax, M. Meuris and M. M. Heyns, Appl. Phys. Lett., 2008, 92(3), 32105.

22 K. Prabhakaran and T. Ogino, Surf. Sci., 1995, 325(3), 263-271.

23 R. Smith and A. Rose, Phys. Rev., 1955, 97(6), 1531-1537.

24 W. J. Yu, S. Y. Lee, S. H. Chae, D. Perello, G. H. Han, M. Yun and Y. H. Lee, Nano Lett., 2011, 11(3), 1344-1350.

25 T.-J. Ha, D. Kiriya, K. Chen and A. Javey, ACS Appl. Mater. Interfaces, 2014, 6(11), 8441-8446.

26 W. Kim, A. Javey, O. Vermesh, Q. Wang, Y. Li and H. Dai, Nano Lett., 2003, 3(2), 193-198.
27 R. Körmer, M. Otto, J. Wu, M. P. M. Jank, L. Frey and W. Peukert, Phys. Status Solidi RRL, 2011, 5(7), 244-246.

28 S. M. George, Chem. Rev., 2010, 110(1), 111-131.

29 M. Simanullang, K. Usami, T. Noguchi, A. Surawijaya, T. Kodera, Y. Kawano and S. Oda, Jpn. J. Appl. Phys., 2014, 53(6S), 06JG04.

30 I. Lokteva, S. Thiemann, F. Gannott and J. Zaumseil, Nanoscale, 2013, 5(10), 4230.

31 C. Mehringer, R. Wagner, T. Jakuttis, B. Butz, E. Spiecker and W. J. Peukert, Aerosol Sci., 2014, 67, 119-130.

32 V. K. Pecharsky and P. Y. Zavalij, Fundamentals of powder diffraction and structural characterization of materials, Springer, New York, 2005. 